\title{
Study on Obtaining High-precision Velocity Parameters of Visual Autonomous Navigation Method Considering Lens Distortion
}

\author{
Han Zhou ${ }^{1, a}$, Qinghua $\mathrm{Li}^{1, \mathrm{~b} *}$ and Hongwei $\mathrm{Li}^{1, \mathrm{c}}$ \\ ${ }^{1}$ Qilu University of Technology, Jinan, China \\ àeredarzh@163.com, 'Iqh@qlu.edu.cn, 'earltrojan@yahoo.com
}

Keywords: Visual navigation; Pyramid LK optical flow method; Lens distortion; Kalman filter

\begin{abstract}
Acquisition of velocity parameter in visual navigation is an important part in autonomous navigation, and its accuracy can directly impact whether this navigation is good or bad. This paper focuses on the study of the velocity parameters accuracy in the pyramid LK optical flow method of visual navigation, introduces the acquisition method of velocity parameters in visual navigation based on pyramid LK optical flow, on the basis of this, corrects the lens distortion, and uses the Kalman filter optimal estimation. The experimental results proves that this method can get a good result and improve the accuracy significantly of velocity parameters.
\end{abstract}

\section{Introduction}

With the rapid development of modern computer technology, high-speed operation, high-reliability, high-precision and other advantages of computers makes the computer technology gradually popularity in all areas of detection and controlling. Visual navigation is an important application of computer vision, for decades, especially in recent years, with the development of optical components, visual navigation has reached a very high precision, autonomous navigation in many occasions has been fully applied. Automation indoor mobile robots have increasingly been used in a wide range of applications [1].

Through the error analysis to traditional pyramid LK optical flow method, two main sources of error are determined:

(1) Systematic error: this error is mainly caused by lens distortion, that is the inherent lens distortion of optical lens, the lens distortion can greatly eliminate by optimizing the production process and the choice of materials, but it cannot completely eliminated[2,3].

(2) random errors: this errors are mainly linear errors, the measured value always contains white Gaussian noise, that is the errors between ideal velocity line and the actual curve line.

This paper aims at these two sources of errors, describes distortion correction algorithm and Kalman filter algorithm, and a series of comparative analysis experiments under different conditions was made.

\section{Introduction of the relevant principles}

Optical flow method is the most commonly used method of visual navigation, the principle is to give each pixel a given velocity vector in a digital image, and forming a motion field, compared with the traditional interframe differentiation algorithm and background differentiation algorithm it is more complex. Classical optical flow algorithm included Horn-Schunck optical flow method and the Lucas-Kanade optical flow method. The pyramid LK optical flow method which we used is a improved algorithm of classic LK optical flow method.

This paper's visual navigation process includes: capture real time video by CCD camera , obtain optical flow between two adjacent frames of a video, transport the velocity of optical flow into the actual velocity, error analysis.

* Corresponding author

Email addresses: lqh@qlu.edu.cn (Qinghua LI) 
Pyramid LK optical flow algorithm. LK optical flow algorithm is a kind of sparse optical flow algorithm, the basic principle is to detect and track feature points. According to the upward-looking camera, we can acquire the image sequences of the terrain. In each set of adjacent images, we select some good features from the first image and record their locations according to the feature detection method [4]. Then, the selected features are tracked to the second image according to the pyramidal implementation of Lucas-Kanade optical flow method [5,6]. Assuming that the image sequence is $I$ $(u, v, n)$, where $(u, v)$ indicates the position of pixels in the image, $\mathrm{n}$ is the order number of a sequence of image frames, to meet the constraint condition of a constant gray level, assuming that the two adjacent grayscale image satisfies the constant constraint:

$$
I(u, v, n)=I(u+d u, v+d v, n+1)
$$

This indicates that the $n+1$ th frame image can be obtained by moving a appropriate vector $d=$ $(d u, d v)^{T}$ of each pixel in the nth frame image, the vector $d$ is called optical flow, which contains the corresponding pixels motion information of interframe.

LK method assumes that all the pixels in a small window within the partial image have the same optical flow, and the value $d$ is estimated obtained by minimized the residuals. Order

$$
\begin{gathered}
J(x)=I(u, v, n) \\
I(x+d)=I(u+d u, v+d v, n+1)
\end{gathered}
$$

The objective function is:

$$
E=\sum_{w}[I(x+d)-J(x)]^{2}
$$

In this formula, $w$ indicates rectangular window, take Taylor series expansion of $I(x+d)$ and then linear approximation to obtain:

$$
I(x+d) \approx I(x)+I^{\prime}(x) d
$$

Obtained the value $\mathrm{d}$ by estimated minimizing $E$, so that $E$ derivative of $d$ is 0

$$
\begin{aligned}
0=\frac{\partial}{\partial d} E & \approx \sum_{w} 2 I^{\prime}(x)\left[I(x)+I^{\prime}(x) d-J(x)\right] \\
D & \approx \frac{\sum_{w} I^{\prime}(x)^{T}[J(x)-I(x)]}{\sum_{w} I^{\prime}(x)^{T} I^{\prime}(x)}
\end{aligned}
$$

When using the LK optical flow algorithm, in order to take account of the feature point tracking both accuracy and robustness, selecting the integration window size is important. In order to solve this problem, we use the pyramid LK optical flow algorithm, which provides a good solution for local tracking of accuracy and robustness. The basic idea of pyramid LK optical flow algorithm is down sampling every digital image interlaced to establish a series of pyramid image level, then beyond this using LK optical flow algorithm. This method can detect the large motion vector in the condition of smaller integration window, this solve both accuracy and robustness issues effectively.

Lens distortion. Lens distortion is the inherent distortion to all optical lens. In practice, it is impossible to completely eliminate, the distortion of the lens can be reduced to a very low level by high levels of production process, but in the edge portions different degrees of deformation will occurs, which the integrated model can be expressed as radial distortion and tangential distortion, it may occur errors or even bring false in the image analysis and processing, so we have to correct the two distortion. 


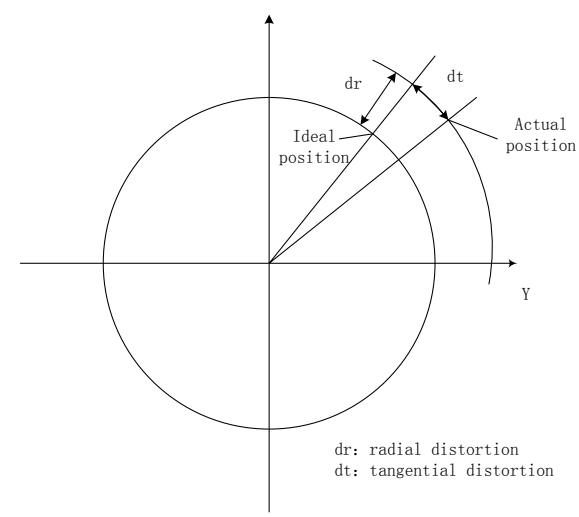

Fig 1. Two kinds of lens distortion

Two kinds of lens distortion are shown in Fig 1:

(1) radial distortion: the vector endpoint changes along the length direction.

(2) tangential distortion: the vector endpoint changes along the tangential direction.

Assuming CCD camera coordinate system $(x, y, z)$ and any point in the ideal image coordinates $\left(X_{u}, Y_{u}\right)$, the focal length is $\mathrm{f}$, then the ideal projective transformation formula:

$$
\begin{aligned}
& X_{u}=f \cdot x / z \\
& Y_{u}=f \cdot y / z
\end{aligned}
$$

$\left(X_{d}, Y_{d}\right)$ indicates the actual image coordinates of the image coordinate system, radial lens distortion and tangential distortion are introduced, its expression:

$$
\begin{aligned}
& X_{u}=X_{d}+k_{1} x\left(x^{2}+y^{2}\right)+\left(p_{1}\left(3 x^{2}+y^{2}\right)+2 p_{2} x y\right) \\
& Y_{u}=Y_{d}+k_{2} y\left(x^{2}+y^{2}\right)+\left(p_{2}\left(x^{2}+3 y^{2}\right)+2 p_{1} x y\right)
\end{aligned}
$$

In this formula $k_{1}, k_{2}$ indicates radial distortion parameters, $p 1$, $p 2$ is the tangential distortion parameters.

Kalman Filter. During the procedure of obtaining velocity parameters in navigation, the error between measurement value and real value is always exists, that is the measured value has noise at any time. Kalman filtering utilization dynamic information of targets, trying to remove the effects of noise, get a good estimate of the target location. This estimate may be estimated on the current target position (filter), it can be estimated for the future location (predicted), it can also estimate the position of the past (interpolation or smoothing). In this paper, the Kalman filter is used to estimate the parameters of the current velocity, that is the velocity measurements filtered and updates of current moment[7,8]. Includes the following steps:

(1) Predicted the current state:

$$
X(k \mid k-1)=A \cdot X(k-1 \mid k-1)+B \cdot U(k)
$$

By the optimal estimate of previous time $X(k-1 \mid k-1)$ and the current control state $U(k)$ predicted the current time state $X(k \mid k-1)$, the test take $U(k)=0$, that means the current control is 0 .

(2) Calculation of covariance matrix:

$$
P(k \mid k-1)=A \cdot P(k-1 \mid k-1) A^{T}+Q
$$

$A^{T}$ is the transpose of the matrix $A, Q$ is the white noise of the system

(3) Reference measurement value estimation:

$$
X(k \mid k)=X(k \mid k-1)+k g(k) \cdot(Z(k)-H \cdot X(k \mid k-1))
$$

$\mathrm{kg}(k)$ is the Kalman gain at time $k, Z(k)$ is the measured value at time $k, H$ is the parameter of measurement system.

(4) Update the data

$$
\begin{gathered}
k g(k)=P(k \mid k-1) \cdot H^{T} /\left(H \cdot P(k \mid k-1) \cdot H^{T}+R\right) \\
P(k \mid k)=(1-k g(k) \cdot H) \cdot P(k \mid k-1)
\end{gathered}
$$

Constantly updated $k g(k)$ and $P(k \mid k)$, and achieve recursive calculations. 


\section{Results analysis}

In this study, the real time avi video is shot by a CCD camera, using MFC dialog to import it and then single-frame image preprocessing.In a constant velocity forward moving navigation vehicle, conducted four experiments:

(1) Pyramid LK optical flow algorithm to obtain the velocity parameter error

(2) Pyramid LK optical flow algorithm + distortion correction obtained velocity parameters error

(3) Pyramid LK optical flow algorithm + Kalman filter obtained velocity parameter error

(4) Pyramid LK optical flow algorithm + Kalman filter + distortion correction obtained velocity parameter error

Four error curves are shown in Fig 2.

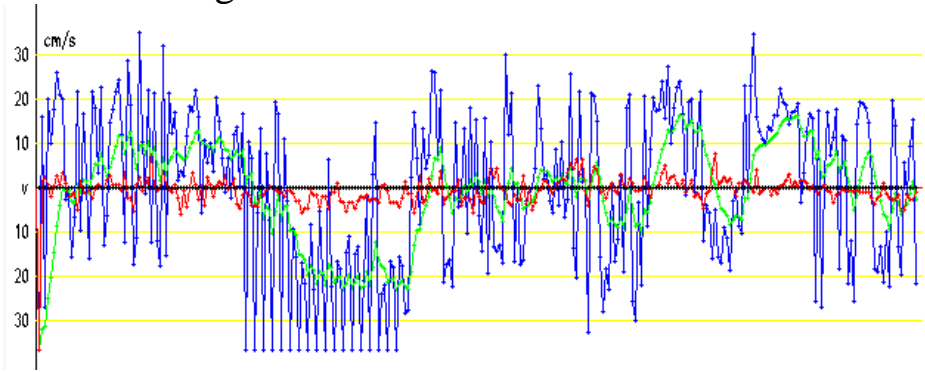

Fig 2. Four curves under the above four cases

Blue curve, red curve, green curve and black curve are experimental results of the above four experiments(1), (2), (3) and (4).

In this experiment, the avi video has 24 frames per second, that is every two pictures are at intervals of $1 / 24 \mathrm{sec}$. In the navigation vehicle which velocity is $100 \mathrm{~cm} / \mathrm{s}$, we take a real-time data analysis every 0.5 seconds:

In Table 1, from e1 to e4 indicates the velocity parameter error of the above four experiments(1), (2), (3) and (4).

Experimental data show: Pyramid LK optical flow algorithm and distortion correction, or a combination with the Kalman filter can significantly improve the velocity error, pyramid LK optical flow algorithm + distortion correction + Kalman filter can obtain the minimal error of velocity.

\begin{tabular}{|c|c|c|c|c|c|}
\hline velocity errors & $0.5 \mathrm{~s}$ & $1.0 \mathrm{~s}$ & $1.5 \mathrm{~s}$ & $2.0 \mathrm{~s}$ & $2.5 \mathrm{~s}$ \\
\hline $\mathrm{e} 1$ & -9.1 & -22.7 & -8.1 & 23.6 & 21.4 \\
\hline $\mathrm{e} 2$ & 0.1 & -1.3 & 1.7 & -1.2 & -1.1 \\
\hline $\mathrm{e} 3$ & 4.0 & -11.6 & 7.6 & 1.9 & 13.5 \\
\hline e4 & -0.0084 & 0.0039 & -0.016 & -0.031 & -0.054 \\
\hline
\end{tabular}

Table 1. Velocity errorsin every 0.5 second under the above four cases [cm/s]

\section{Conclusions}

This paper presents a pyramid LK optical flow algorithm + Kalman filter + distortion correction velocity parameter acquisition methods for visual navigation. Theoretically, this method aims at the main error sources of pyramid LK optical flow algorithm, designs distortion correction and Kalman filter method, has a strong feasibility. Through a series of comparative experiments, this method reduces the error rate by more than $90 \%$, improves the accuracy of the velocity parameter significantly.

\section{Acknowledgement}

This work was supported by the National Natural Science Foundation of China (No.41204025), the National Postdoctoral fund (No.2012M520967) and the Shandong Province Young Scientists Fund (No.BS2013DX031). 


\section{References}

[1] S. J. Kim and B. K. Kim, "Accurate hybrid global self-localization algorithm for indoor mobile robots with two-dimensional isotropic ultrasonic receivers," IEEE Trans. Instrument and Measurement, vol.60, no.10, pp.3391-3404, 2011.

[2] Z.Y. Zhang, S.J. Huang, C. Luo, et al.. Nonlinear distortion correction of camera based on coplanar condition equation [J]. Acta Optica Sinica, 2012, 32(1):0115002.

[3] S. Xu, X.X. Sun, S.G. Liu, et al.. Mode reference approaching method of camera distortion calibration [J]. Acta Optica Sinica, 2013, 33(7):0715001.

[4] J. Shi, C. Tomasi, “Good Features to Track,” in IEEE Int. Conf. Computer Vision and Pattern Recognition., 1994, pp. 593-600.

[5] Pyramidal Implementation of the Lucas Kanade Feature Tracker Description of the algorithm Jean-Yves Bouguet. Intel Corporation Microprocessor Research Labs jean-yves.bouguet@intel.com

[6] S.N Tamgade, V.R. Bora, " Motion Vector Estimation of Video Image by Pyramidal Implementation of Lucas Kanade Optical Flow ”, Second International Conference on Emerging Trends in Engineering\& Technology , 2009.

[7] F. Chen and M.W. Dunnigan, "Comparative study of a sliding-mode observer and Kalman filters for full state estimation in an induction machine," in Proc. IEE Electric Power Applications, vol.149, no.1 pp. 53-64, 2002.

[8] T. Lefebvre, H. Bruyninckx, and J. D. Schutter, "Kalman filters for nonlinear systems: A comparison of performance,” International Journal of Control, vol. 77, no. 7, pp. 639-653, May. 2004. 